Jean Cantelaube, La forge à la catalane dans les

Pyrénées ariégeoises, une industrie à la montagne (XVII ${ }^{e}$ XIX ${ }^{e}$ siècle)

Toulouse, CNRS/Université de Toulouse-Le Mirail, coll. Méridiennes, série « Histoire \& Techniques ", 2005, 805 pages.

\title{
Olivier Codina
}

\section{(2) OpenEdition}

\section{Journals}

Édition électronique

URL : http://journals.openedition.org/dht/1062

DOI : 10.4000/dht.1062

ISSN : 1775-4194

Éditeur :

Centre d'histoire des techniques et de l'environnement du Cnam (CDHTE-Cnam), Société des élèves du CDHTE-Cnam

\section{Édition imprimée}

Date de publication : 1 juin 2008

Pagination : 221-222

ISBN : 978-2-95-30779-1-9

ISSN : 0417-8726

\section{Référence électronique}

Olivier Codina, « Jean Cantelaube, La forge à la catalane dans les Pyrénées ariégeoises, une industrie à la montagne (xVII ${ }^{e}$-xI ${ }^{e}$ siècle) », Documents pour l'histoire des techniques [En ligne], $15 \mid 1^{\text {er }}$ semestre 2008, mis en ligne le 22 octobre 2010, consulté le 22 septembre 2020. URL : http://journals.openedition.org/ dht/1062 ; DOI : https://doi.org/10.4000/dht.1062 


\section{Jean Cantelaube, La forge à la catalane dans les Pyrénées ariégeoises, une industrie à la montagne (XVII ${ }^{e}-\mathrm{XIX}^{e}$ siècle)}

Toulouse, CNRS/Université de Toulouse-Le Mirail, coll. Méridiennes, série « Histoire \& Techniques ", 2005, 805 pages.

\section{Olivier Codina}

\section{RÉFÉRENCE}

Jean Cantelaube, La forge à la catalane dans les Pyrénées ariégeoises, une industrie à la montagne (XVII ${ }^{e}$-XIX ${ }^{e}$ siècle), Toulouse, CNRS/Université de Toulouse-Le Mirail, coll. Méridiennes, série « Histoire \& Techniques », 2005, 805 pages.

1 La forge à la catalane dans les Pyrénées ariégeoises est le texte, amplement remanié, d'une thèse de doctorat brillamment soutenue à l'université de Toulouse-Le Mirail en $2002^{1}$. L'ouvrage de Jean Cantelaube est également la première publication d'une série, inscrite dans la collection Méridiennes, publiée par les Presses Universitaires du Mirail qui a pour titre Histoire \& Techniques. Pour aboutir, cette enquête méticuleuse a demandé la mise en œuvre d'un nombre considérable de fonds documentaires et une profonde connaissance des traités classiques portant sur la sidérurgie directe. Maintes fois décrite, la forge à la catalane a généré une large bibliographie. Cependant, comme le prouvent les résultats de cette enquête, il lui manquait une étude fondée sur une véritable démarche d'historien. En revenant aux sources, en tenant compte des dernières avancées de la recherche sur la réduction directe et en traitant la forge comme un objet historique, l'auteur fait apparaitre bien plus qu'un procédé sidérurgique, il parvient à mettre en lumière le monde à la catalane. 
2 Le livre est structuré en trois grandes parties. Dans un premier temps, la forge est débarrassée de ses topiques. De l'implantation du nouveau procédé au cours du XVII ${ }^{\mathrm{e}}$ siècle, en passant par la naissance et la diffusion de l'appellation à la catalane dans les cercles académiques à partir de la fin du XVIII ${ }^{\mathrm{e}}$ siècle, le mythe à la catalane est mis en évidence. Ce n'est qu'après avoir passé au crible les diverses positions autour de la forge que l'auteur se lance dans la définition de la forge. Machineries, hydraulique, lieux d'implantations et structure des usines livrent une première image de cette industrie. Ce dossier, fondé conjointement sur les textes des ingénieurs des mines et sur les sources d'archives, définit la véritable forge à la catalane: une usine simple, proche parente des moulins, avec un bas foyer caractéristique où les forgeurs mettent en œuvre un savoir-faire spécifique

3 Dans une seconde partie, l'auteur s'attache plus spécialement à l'étude du fonctionnement des ateliers. Au travers des gestes et du vocabulaire, il s'intéresse au rôle des ouvriers. L'un des mérites, et non le moindre, de ce travail est de parvenir à restituer sa place à l'homme dans la châne de production. Le monde sensoriel et l'intelligence des forgeurs sont mis en lumière. Cela permet à Jean Cantelaube de dévoiler les innovations qui se cachaient derrière la notion de routine et de mettre en avant les avantages que présentait ce procédé technique trop souvent décrit comme archaïque. Témoignages contemporains et bilans financiers le prouvent, économe en charbon de bois, la forge donnait des produits compétitifs qui pouvaient concurrencer ceux qui provenaient d'autres filières de production. Cependant, comme cela est démontré, c'est surtout leur qualité, sans cesse renouvelée et améliorée, qui en faisait le renom. La recherche de l'acier dans les produits de la forge, puis la mise en évidence d'une croissante production d'acier, notamment à partir de l'adoption des fours à cémentation vers 1800 , sont deux des dossiers qui permettent de restituer aux fers ariégeois la place de choix qu'ils occupaient au niveau national. La démonstration est claire: loin des notions de routine ou d'immobilisme, les Pyrénées ariégeoises constituent un très bel exemple de continuité technique caractérisée par une succession de savoir-faire renouvelés.

En ce sens, malgré son titre, cette étude ne se limite pas au strict cadre ariégeois. Mineurs, charbonniers et, surtout, forgeurs exportaient leur savoir-faire. Chacun de ces corps de métiers est traité indépendamment et pour chacun d'eux apparaît une zone d'influence qui leur était propre. Ainsi se dessine l'aire à la catalane qui, au-delà des considérations techniques, couvrait une véritable géographie humaine qui englobait le grand ouest pyrénéen et se jouait des frontières politiques. Du Béarn au Roussillon, de la Montagne Noire tarnaise au piémont catalan, ces ouvriers spécialisés moissonnaient des gages qui faisaient progresser favorablement la balance commerciale ariégeoise. L'impact durable de ces migrations saisonnières n'est que l'un des aspects de cette problématique centrée sur le rôle de la sidérurgie dans la communauté. La propriété des feux était détenue majoritairement par la noblesse au XVIII ${ }^{e}$ siècle. Au XIX ${ }^{e}$ siècle, une part non négligeable des édiles régionaux s'implique dans la sidérurgie, quand ils n'appartiennent pas à une famille déjà active dans la métallurgie. Cependant, l'irruption de cette bourgeoisie dans le capital de la sidérurgie de l'Ariège ne doit pas faire oublier un second aspect primordial : la forge était aux mains des paysans (paysans-ouvriers) et elle innervait l'ensemble de la société. Au niveau du département, une partie de la population bénéficiait directement des traitements octroyés par l'importante infrastructure sidérurgique tandis qu'une autre partie était employée 
dans l'une des activités qui en dérivaient. L'organisation des mines, et plus particulièrement celle de Rancié, mais aussi la gestion et l'exploitation de la forêt bénéficient d'un examen minutieux qui conduit à un constat simple: puissance de production, innovation et action structurante, les Pyrénées ariégeoises présentent un véritable modèle industriel.

5 Cependant, les vertus du procédé ne peuvent masquer ses limites. Celles-ci sont abordées dans la troisième partie de l'ouvrage qui s'attache à l'analyse de la disparition de la forge à catalane. Partie intégrante de l'économie montagnarde, ce procédé s'implanta facilement car c'était un modèle de production qui s'adaptait aux besoins de la société dans laquelle il se développait. A contrario, au XIXe siècle, la forge survivait dans une société qui était en voie de paupérisation. L'absence de véritable esprit industriel chez les maîtres de forges ne permit pas l'essor d'une industrie de transformation en accord avec la capacité productive de la région. Malgré certaines différences géographiques ou typologiques, l'étude du marché éclaire l'une des déficiences majeures de ce système: Toulouse monopolisait la diffusion des fers ariégeois et les marchands toulousains ne laissaient que la portion congrue aux maîtres de forges. Au niveau technique, malgré une forte croissance obtenue au cours de la période moderne, le système avait atteint ses limites vers 1830 lorsque la forge à la catalane fut à son optimum. Cependant, les techniciens croyaient toujours à l'avenir du procédé à la catalane et préconisaient des améliorations. L'auteur étudie les diverses solutions techniques proposées par les ingénieurs tout en mettant en exergue l'hostilité qui se faisait jour. La passivité, voire la mauvaise foi, des forgeurs, qui voyaient dans ces tentatives une perte de leur emprise sur la forge, marque une autre des limitations inhérentes au procédé de production. Savoir empirique opposé au savoir scientifique, alors qu'ils avaient adopté les perfectionnements nés dans le monde à la catalane, les forgeurs restèrent réfractaires à la technicité exogène. Ils firent avorter toutes les expériences de modernisation. La crise générale de 1884 vit la fermeture des deux derniers feux à la catalane. En fait, cela faisait déjà une vingtaine d'années qu'ils avaient perdu leur compétitivité face aux productions de masse qu'offraient les nouveaux procédés indirects. L'implantation contemporaine de quelques hautsfourneaux n'y fera rien, le marché sidérurgique ariégeois avait vécu.

En conclusion, il s'agit d'un beau sujet rondement mené qui, en plus, bénéficie d'une écriture claire et fluide. Au niveau régional, cette étude vient utilement s'ajouter aux travaux conduits par Catherine Verna ${ }^{2}$. De la mouline médiévale à la forge à la catalane, ces deux études se complètent et elles permettent de dresser un panorama détaillé de l'un des principaux bassins sidérurgiques du sud de la France. Le livre de Jean Cantelaube constitue un apport d'importance à la bibliographie pyrénéenne. En renouvelant les problématiques et en dépoussiérant le dossier de la forge à la catalane, il a ouvert de nouveaux axes de recherches qui seront utiles aux historiens des Pyrénées mais aussi aux historiens de la technique. 


\section{NOTES}

1. J. Cantelaube, «Forge à la catalane et forgeurs ariégeois. La longue fidélité des Pyrénées à la réduction directe du minerai de fer $\left(\mathrm{XVII}^{\mathrm{e}}\right.$-XIX ${ }^{\mathrm{e}}$ siècle) ", thèse de doctorat, Université de ToulouseLe Mirail, 3 vol. , Toulouse 2002.

2. C. Verna Le temps des moulines. Fer, technique et société dans les Pyrénées centrales (XIII'-XVI ${ }^{e}$ siècle), Paris, Publications de la Sorbonne, 2002.

\section{AUTEURS}

\section{OLIVIER CODINA}

Service de recherche historique du patrimoine culturel d'Andorre 